\title{
An expansion of the field modulus suitable for the description of strong field gradients in axisymmetric magnetic fields: Application to single-sided magnet design, field mapping and STRAFI
}

\author{
Cedric Hugon ${ }^{\mathrm{a}}$, Guy Aubert ${ }^{\mathrm{b}}$, Dimitris Sakellariou ${ }^{\mathrm{c}, *}$ \\ a École Normale Supérieure, Département de Chimie, 24 rue Lhomond, F-75005 Paris, France \\ ${ }^{\mathrm{b}}$ CEA, DSM, IRFU, CEA Saclay F-91191 Gif-sur-Yvette, France \\ ${ }^{\text {C } C E A, ~ D S M, ~ I R A M I S, ~ S I S 2 M, ~ L S D R M ~ C E A ~ S a c l a y ~ F-91191 ~ G i f-s u r-Y v e t t e, ~ F r a n c e ~}$
}

\section{A R T I C L E I N F O}

\section{Article history:}

Received 10 August 2011

Revised 18 October 2011

Available online 9 November 2011

\section{Keywords:}

Portable NMR

Permanent magnets

Static field gradient

Spherical harmonics expansion

1D profiling

Single-sided magnets

Ex situ magnets

STRAFI

Stray field imaging

\begin{abstract}
A B S T R A C T
Mapping (or plotting) the magnetic field has a critical importance for the achievement of the homogeneous magnetic field necessary to standard MR experiments. A powerful tool for this purpose is the Spherical Harmonic Expansion (SHE), which provides a simple way to describe the spatial variations of a field in free space. Well-controlled non-zero spatial variations of the field are critical to MRI. The resolution of the image is directly related to the strength of the gradient used to encode space. As a result, it is desirable to have strong variations of the field. In that case, the SHE cannot be used as is, because the field modulus variations are affected by the variations of all components of the field. In this paper, we propose a method based on the SHE to characterize such variations, theoretically and experimentally, in the limit of an axisymmetric magnetic field. Practical applications of this method are proposed through the examples of single-sided magnet design and characterization, along with Stray-Field Imaging (STRAFI).
\end{abstract}

(c) 2011 Elsevier Inc. All rights reserved.

\section{Introduction}

Nuclear Magnetic Resonance (NMR) is a very versatile technique with broad and diverse fields of application ranging from protein structure analysis (through spectroscopy) to medical imaging (MRI). It requires a complicated instrumentation involving a spectrometer, RF components, RF amplifiers, coils or antennas, and a magnet providing a static magnetic field. This magnet is critical as the requirements on the static magnetic field are extremely stringent. The field must indeed be controlled spatially (homogeneity) and temporally (limited field drift) to an extreme precision, usually ppb (part per billion) for spectroscopy and ppm (part per million) for imaging. The design of such a magnet can be done most efficiently using the Spherical Harmonic Expansion (SHE) to describe the spatial variations of the main component of the field. This technique was first extensively described for coil systems in the 1980s [1] and further developed in the context of permanent magnets lately [2,3]. This is not an intuitive approach as NMR is sensitive to the magnitude of the field, $|\vec{B}|$, which cannot be

\footnotetext{
* Corresponding author.

E-mail addresses: cedric.hugon@ens.fr (C. Hugon), guy.aubert@cea.fr (G. Aubert), dsakellariou@cea.fr (D. Sakellariou).
}

described by a SHE ( $|\vec{B}|$ does not satisfy the Laplace equation). However, as long as the field variations are small compared to its nominal value, the modulus can be considered dominated by the variations of the main component of the field, usually named $B_{z}$ and found at the origin, defined here as the center of the Region of Interest (RoI). Thus, the SHE approach is an approximation where only one cartesian component of the field is considered. But it is highly accurate when dealing with homogeneous fields. Therefore, it becomes inappropriate when dealing with rapid spatial variations of the field. While NMR always requires a very homogeneous background field, MRI makes use of superimposed field "gradients" to encode the space. ${ }^{1}$ As a result, three gradients are necessary to obtain 3D images. The best achievable resolution is roughly given by $\frac{2}{\gamma G T_{2}}$, where $G$ is the applied field gradient, $T_{2}$ is the transverse relaxation time of the observed nucleus, and $\gamma$ is its gyromagnetic ratio [4]. $T_{2}$ is intrinsic to the object to be observed and cannot be changed. ${ }^{2}$ The increase of resolution can therefore be obtained only through an increase of the gradient strength. This

\footnotetext{
${ }^{1}$ In this case, the word "gradient" refers to a well-controlled linear variation of the field modulus in a given direction of space. While non-linear variations can be used for the encoding, linear ones are often preferred.

${ }^{2}$ In many cases, the resolution will be limited by $T_{2}^{*}$ and not $T_{2}$. However, $T_{2}^{*}$ can be modified by various techniques [4] but $T_{2}$ remains the absolute limit.
} 
is one of the goals of stray-field imaging (STRAFI), where the sample is placed in the stray-field of a powerful NMR magnet so that field gradients of the order of $10-100 \mathrm{~T} \mathrm{~m}^{-1}$ can be obtained [5]. Intense gradients can also be obtained with single-sided permanent magnets [6-9]. In such cases, the SHE approximation cannot be used anymore: the field modulus variations are not dominated by only one Cartesian component but involve all of them. This issue is well known in MRI under the name "concomitant fields (or gradients)" [10], or "Maxwell terms".

This problem has been the object of diverse studies, trying to cancel out the deleterious effect of these mandatory imperfections induced by the gradient systems [11-13], or hardware systems compensating these fields $[14,15]$. However, to our knowledge, nothing has been proposed to describe the variations of the modulus in a condensed fashion similar to the SHE, in order to perform efficient field mapping, and efficient design of the gradient-generating system. We introduce in this paper a general theoretical framework to describe a strong, axially symmetric, gradient of field. This approach is focused on the modulus of the field. We then apply this method to the design and characterization of a singlesided permanent magnet. Finally, we present a few elements based on this description in the context of STRAFI.

\section{The origin of concomitant fields, or Maxwell terms}

While the physics underlying concomitant fields have been covered many times in the past $[10,11]$, it is worthwhile to start from the beginning. These so-called "Maxwell terms" are simply due to the Maxwell's equations imposing relationships between the spatial derivatives of the magnetic field. We have indeed in free space

$\vec{\nabla} \times \vec{B}=0, \quad \vec{\nabla} \cdot \vec{B}=0$,

which implies for the Cartesian components the following relations:

$$
\begin{array}{ll}
\frac{\partial B_{z}}{\partial y}=\frac{\partial B_{y}}{\partial z}, & \frac{\partial B_{x}}{\partial z}=\frac{\partial B_{z}}{\partial x}, \\
\frac{\partial B_{y}}{\partial x}=\frac{\partial B_{x}}{\partial y}, & \frac{\partial B_{x}}{\partial x}=-\frac{\partial B_{y}}{\partial y}-\frac{\partial B_{z}}{\partial z} .
\end{array}
$$

The first-order field variations can be written as

$\vec{B}(x, y, z)=\left[\begin{array}{ccc}\frac{\partial B_{x}}{\partial x} & \frac{\partial B_{x}}{\partial y} & \frac{\partial B_{x}}{\partial z} \\ \frac{\partial B_{y}}{\partial x} & \frac{\partial B_{y}}{\partial y} & \frac{\partial B_{y}}{\partial z} \\ \frac{\partial B_{z}}{\partial x} & \frac{\partial B_{z}}{\partial y} & \frac{\partial B_{z}}{\partial z}\end{array}\right]\left[\begin{array}{l}x \\ y \\ z\end{array}\right]+O\left(\vec{r}^{2}\right)$.

Using the previous relations we can note ${ }^{3} G_{x}=\frac{\partial B_{z}}{\partial x}, G_{y}=\frac{\partial B_{z}}{\partial y}$, $G_{z}=\frac{\partial B_{z}}{\partial z}, g=\frac{\partial B_{y}}{\partial x}=\frac{\partial B_{x}}{\partial y}$ and setting $-\alpha G_{z}=\frac{\partial B_{x}}{\partial x}$, we have

$\vec{B}(x, y, z)=\left[\begin{array}{ccc}-\alpha G_{z} & g & G_{x} \\ g & (\alpha-1) G_{z} & G_{y} \\ G_{x} & G_{y} & G_{z}\end{array}\right]\left[\begin{array}{l}x \\ y \\ z\end{array}\right]+O\left(\vec{r}^{2}\right)$.

This last equation shows clearly the inevitable coexistence of gradients of the different Cartesian components. The effect of these gradients in the modulus can be assessed by the following considerations. If we consider the field $\vec{B}_{0}$ at the origin (center of the RoI), we can set $\vec{B}_{0}=B_{0} \vec{u}_{z}$. Let us now consider a small perturbation of the field $\vec{b}(x, y, z)$ which satisfies the previous discussion, implying that its components are all of the same order of magnitude. We can write

$\vec{B}(x, y, z)=\vec{B}_{0}+\vec{b}(x, y, z)$,

which yields for the modulus

\footnotetext{
${ }^{3}$ We use here the usual notations in MRI [11]. Another description can be found in [16].
}

$B(x, y, z)=B_{0} \sqrt{1+2 \frac{b_{z}}{B_{0}}+\frac{\vec{b}^{2}}{B_{0}^{2}}}$.

Expanding in Taylor series gives

$B(x, y, z)=B_{0}\left(1+2 \frac{b_{z}}{B_{0}}+O\left(\frac{\vec{b}^{2}}{B_{0}^{2}}\right)\right)$,

which shows that when the three components of $\vec{b}$ are of the same order of magnitude, the one along the main field dominates (i.e. the main field truncates the other components). Thus, when we consider a field along $O z$, we only have to care about the third line in the tensor seen in Eq. (2). However, if the gradient strength and the extent of the object are sufficient (i.e. we do not have $\left.B_{0} \gg \Delta B=\vec{G} \cdot \vec{r}\right)$, it is necessary to consider all components.

In the case of homogeneous fields, the field is dominated by the main component, so that the design and characterization of the field profile can be done using the SHE of the main component. It is not as straightforward when we start considering a strong gradient.

\section{SHE-based framework for axially-symmetric strong 1D gradients}

This section focuses on results published earlier in $[9,17]$ and presented here for convenience. We consider here a situation where the distribution of field sources (currents, poles) is axially symmetric. In that case, it is obvious that everywhere in space, the field can only be radial or longitudinal, or a combination of both. For convenience, we can express the magnetic field in cylindrical coordinates $(\rho, \phi, z)$, with its two components $B_{\rho}$ and $B_{z}$. Following the notation we introduced in $[2,3]$ and using spherical coordinates $(r, \theta, \phi)$ for the calculation point, we can write the general form of the SHE of $B_{z}$ as

$B_{z}=Z_{0}+\sum_{n=1}^{\infty} Z_{n} r^{n} P_{n}(\cos \theta)$

It is also possible to show (see Appendix A) that $B_{\rho}$ can be written as the following expansion

$B_{\rho}=\sum_{n=1}^{\infty}-\frac{1}{n+1} Z_{n} r^{n} P_{n}^{1}(\cos \theta)$

This result shows once more how the different components of the magnetic field are linked: in free space, the knowledge of one component is sufficient to gain knowledge of all of them. We can thus derive the full spatial variation of the modulus of the field based on the SHE of one component. To simplify calculations, we will work with $B^{2}$ instead of the modulus. We have indeed

$|\vec{B}|^{2}=B_{\rho}^{2}+B_{z}^{2}=R_{0}+\sum_{n=1}^{\infty} R_{n}(\theta) r^{n}$,

where $R_{n}(\theta)$ can be obtained based on the expansion of $B_{\rho}$ and $B_{z}$. As a result, the square of the modulus of the field can be expressed only based on the terms of the SHE of $B_{z}$.

The $R_{n}$ terms are not of great interest as their expression becomes rapidly complicated when $n$ increases, and do not provide an intuitive description of the field variations. It seems preferable to convert the expansion in Eq. (8) to an expansion in cylindrical coordinates. It is simple matter to carry out this transformation and obtain

$|\vec{B}|^{2}=\sum_{n=0}^{\infty} \sum_{m=0}^{\infty} S_{n}^{m} \rho^{m} z^{n}$ 
where $S_{n}^{m}$ can be obtained by transforming the $R_{n}$ terms. We can give a few $S_{n}^{m}$ as only because of the $S_{0}^{2}$ term that the field is not uniform in the transverse direction.
$S_{n}^{m}=\left\{\begin{array}{ccc}n \mid m & 0 & 2 \\ 0 & Z_{0}^{2} & \frac{1}{4} Z_{1}^{2}-Z_{0} Z_{2} \\ 1 & 2 Z_{0} Z_{1} & -3 Z_{0} Z_{3} \\ 2 & Z_{1}^{2}+2 Z_{0} Z_{2} & -\frac{3}{2} Z_{1} Z_{3}-6 Z_{0} Z_{4} \\ 3 & 2\left(Z_{1} Z_{2}+Z_{0} Z_{3}\right) & -\left(Z_{2} Z_{3}+4 Z_{1} Z_{4}+10 Z_{0} Z_{5}\right) \\ 4 & Z_{2}^{2}+2\left(Z_{1} Z_{3}+Z_{0} Z_{4}\right) & -\left(\frac{3}{4} Z_{3}^{2}+3 Z_{2} Z_{4}+\frac{15}{2} Z_{1} Z_{5}+15 Z_{0} Z_{6}\right)\end{array}\right.$

All odd terms in $\rho$ are null due to symmetry.

It is in fact possible to establish analytical formulae giving directly any $S_{n}^{m}$ terms. While the general equation is quite heavy and difficult to use, we can give here formulae for special cases, namely $S_{n}^{0}$ and $S_{0}^{2 m, m \geqslant 1}$. We have indeed

$S_{0}^{0}=Z_{0}^{2}$,

$S_{n}^{0}=2 Z_{0} Z_{2}+\sum_{j=1}^{n-1} Z_{j} Z_{n-j}$,

$$
\begin{aligned}
(-1)^{m} S_{0}^{2 m, m \geqslant 1}= & 2 Z_{0} Z_{2 m} \frac{(2 m-1) ! !}{(2 m) ! !} \\
& -\sum_{q=1}^{m} Z_{2 q-1} Z_{2 m-2 q+1} \frac{(2 q-1) ! !(2 m-2 q+1) ! !}{(2 q) ! !(2 m-2 q+2) ! !} \\
& +\sum_{q=1}^{m-1} Z_{2 q} Z_{2 m-2 q} \frac{(2 q-1) ! !(2 m-2 q-1) ! !}{(2 q) ! !(2 m-2 q) ! !}
\end{aligned}
$$

$$
\begin{aligned}
(-1)^{m} S_{1}^{2 m, m \geqslant 1} & =2 Z_{0} Z_{2 m+1} \frac{(2 m+1) ! !}{(2 m) ! !} \\
& +\sum_{q=1}^{m}\left(Z_{2 q} Z_{2 m-2 q+1}-Z_{2 q-1} Z_{2 m-2 q+2}\right) \\
& \times \frac{(m-2 q+1)(2 q-1) ! !(2 m-2 q+1) ! !}{(m-q+1)(2 q) ! !(2 m-2 q) ! !} .
\end{aligned}
$$

The great advantage of such an expansion is that it involves only terms of the SHE of $B_{z}$ and is completely adapted to a separate treatment of the longitudinal and transverse directions. In addition, the $S_{n}^{m}$ matrix shows right away that it is strictly impossible to achieve a "perfect" gradient of the field strength. We mean by "perfect", a pure linear variation of the modulus along one direction and no variations in other directions over an extended volume. For example, $S_{2}^{0}$ and $S_{0}^{2}$ involve $Z_{0}, Z_{1}$, and $Z_{2}$ in an independent manner, so that both cannot be canceled if $Z_{1} \neq 0$. This results in either a quadratic variation of the field in the radial direction, or a quadratic variation in the longitudinal direction, superimposed over the gradient.

We can identify two main types of useful field profiles featuring a strong gradient. One consists in a perfectly linear variation of the field along $\mathrm{Oz}$, tolerating significant variations of the field in the transverse direction. We will call this a "straight" gradient. Such a profile can be useful when dealing with samples with very small lateral extent but requiring very high resolution.

In that case, we can simply work with $B_{z}$ only and obtain a highly linear variation of the field on-axis by canceling as many terms other than $Z_{0}$ nor $Z_{1}$ as possible. It is interesting to note that this implies the cancelation of all terms in the $S_{n}^{m}$ matrix, but $S_{0}^{0}, S_{1}^{0}, S_{2}^{0}, S_{0}^{1}$, and $S_{0}^{2}$. In the perfect case (all $Z_{n \neq 0,1}$ canceled), it is

$$
\left.\begin{array}{c}
4 \\
\frac{1}{4} Z_{2}^{2}-\frac{3}{8} Z_{1} Z_{3}+\frac{3}{4} Z_{0} Z_{4} \\
\frac{3}{4}\left(Z_{2} Z_{3}-Z_{1} Z_{4}+5 Z_{0} Z_{5}\right) \\
\frac{3}{4}\left(Z_{2} Z_{4}+15 Z_{0} Z_{6}+\frac{3}{2} Z_{3}^{2}\right) \\
\frac{5}{4}\left(Z_{2} Z_{5}+3 Z_{3} Z_{4}+3 Z_{1} Z_{6}+21 Z_{0} Z_{7}\right) \\
\frac{15}{4}\left(Z_{4}^{2}+Z_{2} Z_{6}+\frac{3}{2} Z_{3} Z_{5}+\frac{7}{2} Z_{1} Z_{7}+14 Z_{0} Z_{8}\right)
\end{array}\right\}
$$

The other type of profile privileges the uniformity of the field in the transverse direction. We will call such a profile a "flat" gradient. In that case, we need to consider the $S_{n}^{m}$ matrix. The priority is to cancel the $S_{0}^{m}$ coefficients. This implies undesired values of the $S_{n}^{0}$ coefficients, resulting in a non-linear variation of the field along the axis. Cancellation of cross-terms such as $S_{1}^{2}, S_{1}^{4}$ and others might also be obtained in the process. We can give the exact values of the first SHE terms of $B_{z}$ necessary to provide such a field profile. These values can be given in terms of the desired field strength $\left(Z_{0}\right)$ and the desired gradient strength $\left(Z_{1}\right)$ :

$$
\begin{aligned}
& Z_{2}=\frac{1}{4} \frac{Z_{1}^{2}}{Z_{0}}, \\
& Z_{3}=0, \\
& Z_{4}=-\frac{1}{48} \frac{Z_{1}^{4}}{Z_{0}^{3}}, \\
& Z_{5}=0 .
\end{aligned}
$$

As usual, conditions on the lower degrees should be satisfied in priority. Conditions on higher degree terms can be obtained in the same manner but were not written here for the sake of space. The control of higher degrees allows to increase the extent of the uniform region in the transverse direction but also increases the extent along $\mathrm{Oz}$ where the isofields are "flat". These results were given in the past [17] and the condition on $Z_{2}$ was more recently proposed by an independent team [18]. In most cases, the distortion introduced by the non-linearity of the variation of the field along $\mathrm{Oz}$ is tolerable, because the strong gradient and the bandwidth of the NMR receptor limits the extent of the observable sample.

We think this mathematical framework can be useful in many cases and give three examples in the following section. As the term "field gradient" may be confusing, we would like to advise the reader that, through the rest of this paper, this term refers to the desired 1D linear spatial variation of the field magnitude.

\section{Application of the $S_{n}^{m}$ framework}

\subsection{Single-sided magnet design and shimming}

A common drawback of single-sided systems is that the field profile usually displays a very strong field gradient (anywhere between 1 and $20 \mathrm{~T} \mathrm{~m}^{-1}$ ). It is of course possible to achieve a highly homogeneous field in a given region but the price to pay is very high in field strength [17]. Nevertheless, strong field gradients are still of interest in various applications of NMR, for example STRAFI, diffusion measurements, etc. However, one difficulty is to have a "clean" field profile, with specific properties, such as an 
extremely constant gradient in a given region, or a uniform field in directions transverse to the gradient. The discussion of the previous section, especially Eqs. 10 and 15, provides a direct mean to design an axially symmetric single-sided magnet (based on coils or permanent magnets) with a well-controlled field gradient. While the usual method based on spherical harmonics $[1,3,17]$ consists in canceling as many terms as possible to achieve homogeneity, we will need here to get as many terms as possible to satisfy the conditions provided in Eq. (15).

Because of their portability, permanent magnets are often chosen to realize single-sided systems [7,9,19-21]. However, no real systematic approach has been proposed until now to design such magnet systems. We propose here to use the preceding framework along with a building-block approach to devise axially symmetric structures providing the desired field profile.

We define elementary magnet blocks from which we will compose the final magnet system. A simple block is the hollow cylinder magnetized along its axis. This hollow cylinder has its axis on $O z$, features an inner radius $a_{1}$ and an outer radius $a_{2}$ and is delimited by two planes of elevation $b_{1}$ and $b_{2}$ with $b_{1}<b_{2}$. The field generated by such a block can be determined analytically in the entire space [22]. We can even go further by determining analytically the SHE terms of such a block. Following the demonstration of Appendix B also presented earlier in [17], we find

$$
\begin{aligned}
& Z_{0}=\frac{\mu_{0} M}{2}\left[\left[\frac{b}{\sqrt{a^{2}+b^{2}}}\right]_{a_{1}}^{a_{2}}\right]_{b_{1}}^{b_{2}}=\frac{\mu_{0} M}{2}\left[[\cos \alpha]_{a_{1}}^{a_{2}}\right]_{b_{1}}^{b_{2}}, \\
& Z_{n \geqslant 1}=-\frac{\mu_{0} M}{2 n}\left[\left[\frac{1}{c^{n}} \sin \alpha P_{n}^{1}(\cos \alpha)\right]_{a_{1}}^{a_{2}}\right]_{b_{1}}^{b_{2}},
\end{aligned}
$$

with

$a=c \sin \alpha$,

$b=c \cos \alpha$,

$c=\sqrt{a^{2}+b^{2}}$.

The brackets $[f(s)]_{s_{1}}^{s_{2}}$ are a short notation for $f\left(s_{2}\right)-f\left(s_{1}\right)$.

Based on these relations, it is "only" a matter of non-linear optimization to find the correct parameters for a proper number of rings ( $n+1$ to control $n$ terms) and satisfy the different conditions. We have designed magnet systems based on these considerations generating gradient and homogeneous profiles [9,17]. Based on these designs, we realized one prototype of a magnet $(20 \mathrm{~cm}$ diameter, $12 \mathrm{~cm}$ height, $35 \mathrm{~kg}$ ) generating $0.3 \mathrm{~T}$ in the region of interest located $2 \mathrm{~cm}$ above the surface of the magnet. The gradient strength was $3.3 \mathrm{~T} \mathrm{~m}^{-1}$. While we have assumed until now that the magnet blocks are perfect, the actual system features numerous imperfections, which can be assessed statistically. Such a sensitivity analysis was performed and described in [17] and will be published in a subsequent paper. The outcome of this analysis showed that a properly designed magnet can be expected to behave better than a simple magnet cylinder, despite imperfections. The analysis of a shimming system was also performed. This shim system was built in the magnet by design, leaving some parts of the magnet adjustable. These parts are assembled at the theoretical position and slid radially to adjust the magnet (see Fig. 1). Shimming is done based on the measurements provided by the plotting method described in Section 4.2.

This shimming procedure achieved high uniformity in transverse planes ( $\Delta B<100 \mathrm{ppm}$ over $1 \mathrm{~cm}$ diameter). Imaging of thin slices of olive oil with a resolution of about $20 \mu \mathrm{m}$ was possible [9]. This resolution figure was obtained by computing the Point Spread Function (PSF), as the derivative of the image of a sharp edge. The FWHM of the PSF gives the instrument resolution (see Fig. 2).

\subsection{Field plotting of strong gradients using an NMR probe}

We consider now a situation where we expect to find an axially symmetric field gradient with a well-determined profile (for example uniform in transverse planes). The magnet producing this field profile is imperfect and needs to be adjusted, or shimmed. This requires to characterize the field profile first. This is a well-known problem in the design of homogeneous fields, where the field magnitude is dominated by the main component (called here $B_{z}$ ). It is usually necessary to retrieve the SHE terms $Z_{n}, X_{n}^{m}$ and $Y_{n}^{m}$ found in the following general equation for the field in free space $[3,17]$ :

$$
\begin{aligned}
& B_{z}(r, \theta, \phi)=Z_{0} \\
& +\sum_{n=0}^{\infty} r^{n}\left(Z_{n} P_{n}(\cos \theta)+\sum_{m=1}^{\infty}\left(X_{n}^{m} \cos m \phi+Y_{n}^{m} \sin m \phi\right) P_{n}^{m}(\cos \theta)\right) .
\end{aligned}
$$

The general method to tackle this problem is well-known $[1,3,17]$ and consists in measuring the field modulus (equivalent to $B_{z}$ ) in well-chosen points with a high precision typically in the range of the ppm or better (usually, NMR itself is used to perform these measurements). The SHE terms are then extracted from the set of field values using discrete Fourier transform and polynomial interpolation.

In the context of an axially symmetric strong gradient, we have shown in Section 3 that the SHE of $B_{z}$ is still sufficient to describe the field modulus and control it. However, the measurement procedure cannot be the same as we do not have $B_{z} \approx|B|$ anymore. We show in this section how we can still access $B_{z}$ with reasonable precision, using NMR measurements.

Following Maxwell's equations, we have

$$
\vec{\nabla} \cdot \vec{B}=0 \text {. }
$$

In a rotationally symmetric system and in cylindrical coordinates $(\rho, \phi, z)$, we have $\frac{\partial B_{\phi}}{\partial \phi}=0$ and thus,

$\frac{1}{\rho} \frac{\partial\left(\rho B_{\rho}\right)}{\partial \rho}=-\frac{\partial B_{z}}{\partial z}$

which can be solved to obtain

$\frac{\partial B_{\rho}}{\partial \rho}=-\frac{1}{2} \frac{\partial B_{z}}{\partial z}$

or,

$B_{\rho}(\rho)=-\frac{1}{2} \frac{\partial B_{z}}{\partial z} \rho$

We can use Eq. (23) to compensate NMR measurements and obtain the value of $B_{z}$. We have indeed at a point of coordinate $(\rho, \phi$ $, z)$,

$|\vec{B}|=B=\sqrt{B_{z}^{2}+B_{\rho}^{2}}$.

As the sign of $B_{z}$ does not change in the region of measurement, we can write

$B_{z}=\sqrt{B^{2}-B_{\rho}^{2}}$.

Measurements along $O z$ are unaffected by $B_{\rho}$, so that we can compute the axial SHE terms $\left(Z_{n}\right)$ of $B_{z}$. Thus, we can compute accurately $\frac{\partial B_{z}}{\partial z}$ on axis. Assuming this quantity does not vary much over the measurement volume, we can compute $B_{\rho}$ in any measurement point with Eq. (23) and convert into $B_{z}$ the field measured by NMR through Eq. (25). Following this, we will be able to extract all SHE terms of $B_{z}$ using the usual measurement scheme [1,3]. It is 


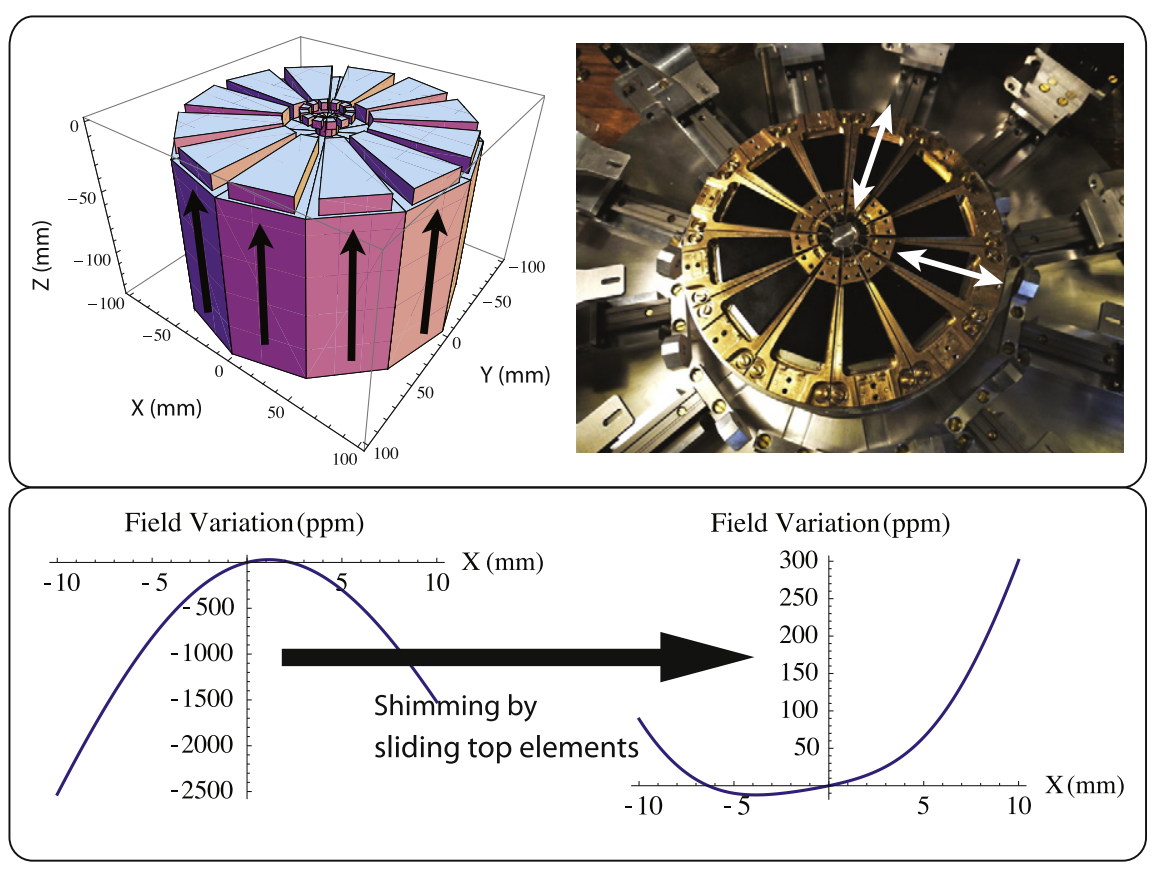

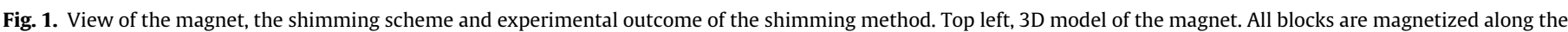

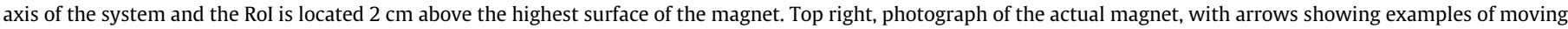

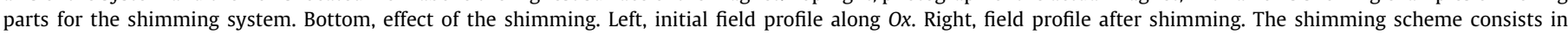

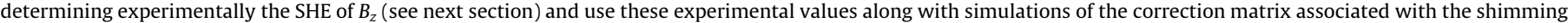
system. Simple calculations yield the necessary displacements of the different mobile parts. The final result is obtained in three iterations (less than $2 \mathrm{~h}$ ).

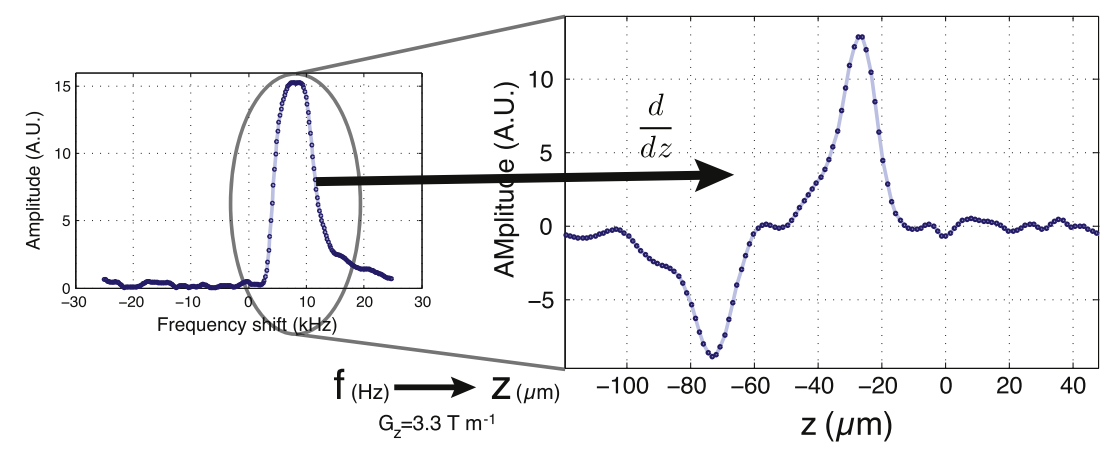

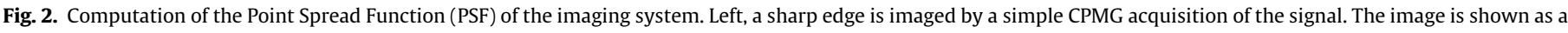

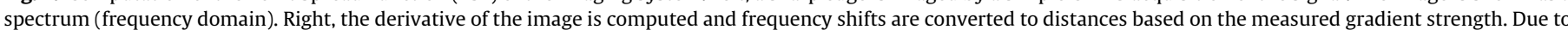

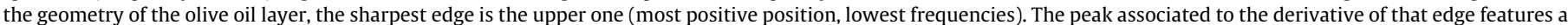
$20 \mu \mathrm{m}$ FWHM.

however necessary to assess the error made on the value of $B_{z}$ with this approximation. We can distinguish the error $\Delta^{B} B_{z}$ due to uncertainties $\Delta B$ on $B$, and the error $\Delta^{B_{\rho}} B_{z}$ due to uncertainties $\Delta B_{\rho}$ in $B_{\rho}$.

$\Delta^{B} B_{z}=\frac{B}{B_{z}} \Delta B$

$\Delta^{B_{\rho}} B_{z}=\frac{B_{\rho}}{B_{z}} \Delta B_{\rho}$.

In most cases, $\frac{B}{B_{2}} \approx 1$. Considering the field generated by the magnet presented in Section 4.1, we can give an example of error estimation. We have for a radius $r=6 \mathrm{~mm}, \frac{B_{\rho}}{B_{z}} \approx 0.05 . \Delta B_{\rho}$ is given by the difference between the actual $B_{\rho}$ and the one we predict based on the gradient on-axis, using $B_{\rho}=-\frac{1}{2} \frac{\partial B_{z}}{\partial z} \rho$. In the case of a perfectly axisymmetric magnet, this difference can be estimated to be less than $2.5 \mathrm{ppm}$ of the nominal $B_{z}$ at a radius of $6 \mathrm{~mm}$. Thus, we have
$\Delta^{B_{\rho}} B_{z}=0.125 \mathrm{ppm}$. The total uncertainty on the calculated $B_{z}$ being given by $\sqrt{\left(\Delta^{B} B_{z}\right)^{2}+\left(\Delta^{B_{\rho}} B_{z}\right)^{2}}$, if we assume the uncertainty on $B$ (the linewidth of the signal) is greater than $\pm 1 \mathrm{ppm}$, we have $\Delta B_{z} \approx \Delta B$. Thus, for a perfect magnet, a $B_{z}$ SHE calculation based on such measurements features the same precision as for a homogeneous magnet with the same NMR signal linewidth. ${ }^{4}$

Until now, we have assumed the field profile perfectly axially symmetric. However, as we said at the beginning of this section, the magnet will feature imperfections and prove wrong the symmetry assumption. This induces larger discrepancies between the actual $B_{z}$ and the calculated one, with a high dependence on the

\footnotetext{
4 This first-degree correction is sufficient because transverse terms enter only in second order in the modulus. In some extreme gradient cases, it might be necessary to use higher degree corrections in the calculation, which is straightforward.
} 

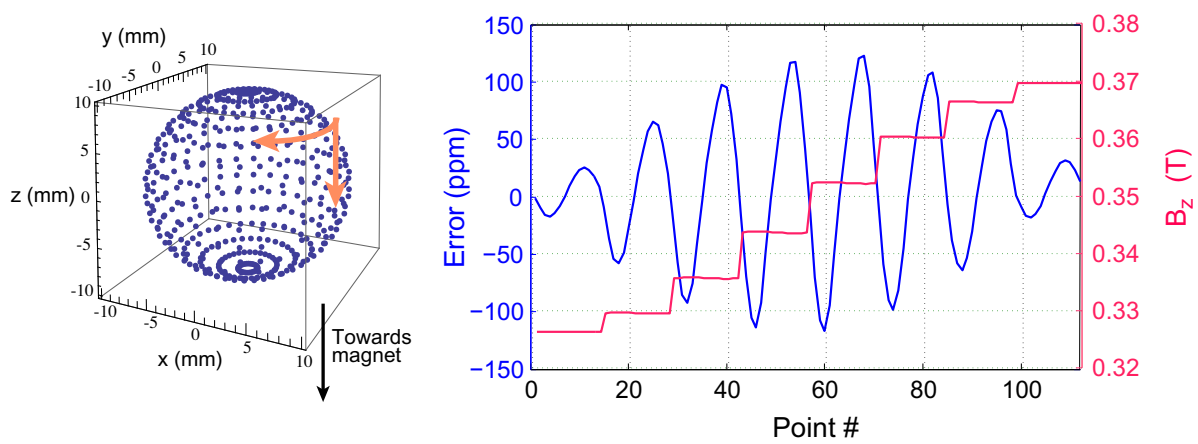

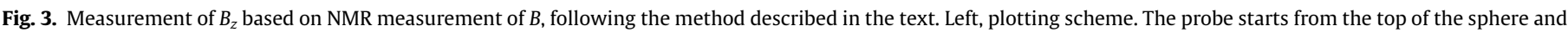

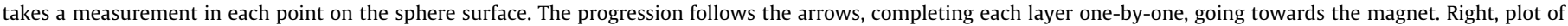
simulated measured $B_{z}$ values, along with the error made, for an imperfect, realistic, magnet. The maximum error is about 100 ppm.

field profile. This can be explained through the possible compensation of variations of $B_{z}$ by variations of $B_{\rho}$ in the modulus value seen by the NMR probe. $B_{\phi}$ is negligible as inhomogeneities in $\phi$ are expected to be $<1000 \mathrm{ppm}$ (we assume that axial symmetry is reasonably realized). Simulations based on the magnet of the previous section show that for this extremity, the maximum error on $B_{z}$ is about $100 \mathrm{ppm}$ (see Fig. 3). The average deviation from the mean error is about $35 \mathrm{ppm}$. Of course, if the field profile has a better axial symmetry (less variations in $\phi$ ), we gain accuracy and precision (less offset and smaller standard deviation). These errors are not random and their effect on SHE term measurements is not equivalent to the effect of a field noise with a matching standard deviation. It is thus important to have an idea of the field profile before plotting (through simulations).

Finally, a good way of estimating the relevance of such measurements is to compare an experimental field map with a simulated one, based on the extracted SHE terms. Taking the magnet of Section 4.1 as an experimental example, we performed both a Cartesian field map and a SHE measurement, using a small NMR probe providing a spectral resolution of $20 \mathrm{ppm}$ [17]. We were able to retrieve reliably all $Z_{n}, X_{n}^{m}$ and $Y_{n}^{m}$ terms up to degree four, which were sufficient to describe the field in the RoI. Fig. 4 shows a comparison between the experimental field profile in a transverse plane obtained from the Cartesian measurements, and the reconstructed from the measured SHE terms. The agreement is excellent, proving the SHE measurements suitable as input values for the shimming of the magnet. This allowed the accomplishment of the performances shown in the previous section and previous publications $[9,17]$.

Thus, we have here a means of retrieving $B_{z}$ from $B$ with high precision in the context of a field profile close to axial symmetry. We can assess through SHE analysis the deviation from axial symmetry and correct it by compensating the $X_{n}^{m}$ and $Y_{n}^{m}$ terms, while the $Z_{n}$ terms can be used in the framework of Section 3 to charac-
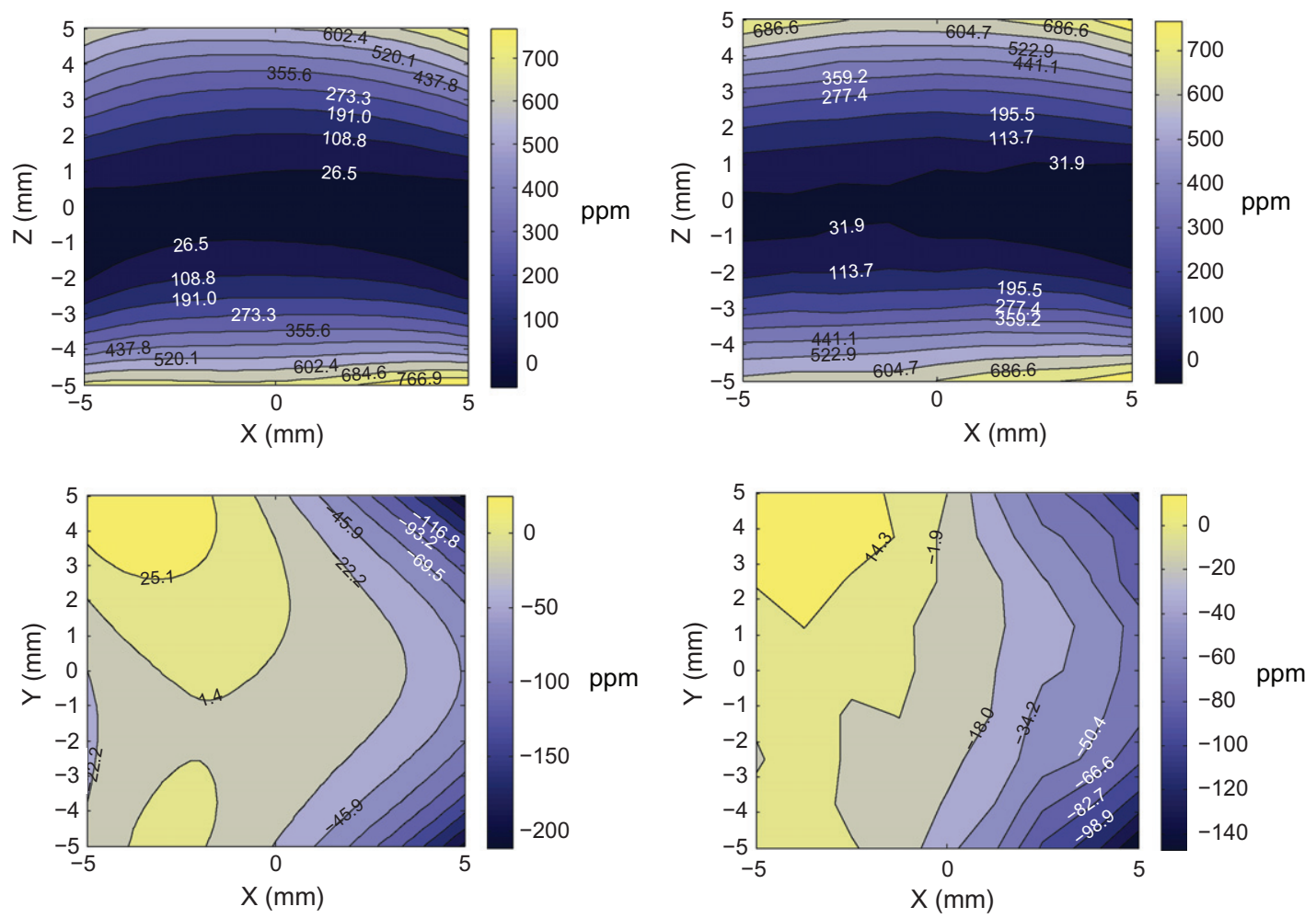

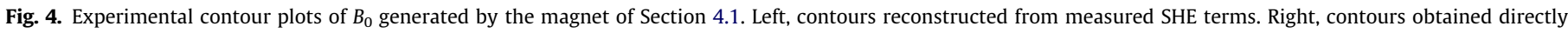

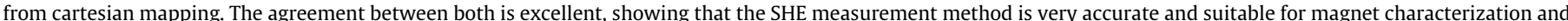

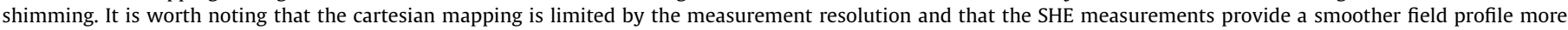
suitable for NMR simulations. 
terize the gradient profile and possibly shim the system to obtain the expected profile.

\subsection{Stray Field Imaging (STRAFI) NMR}

A specific case where the discussion of Section 3 can be found useful is STRAFI NMR [5]. While several studies provided a lot of insight to the NMR aspects of STRAFI [23-25], it seems to us that few have focused on the understanding of the behavior of the magnetic field in this context. A clear view of this aspect is crucial and can lead to hardware improvement for this technique.

In most cases, a STRAFI experiment is conducted in the fringe field of a superconducting magnet (usually axially symmetric), and consists in placing a NMR probe at a location away from the center of the magnet, but still on the axis. As a result, the magnetic field points along $\mathrm{O} z$ at the center of the new region of interest. We can thus still use an expansion of the form of Eq. (6) for the field on-axis. However, the strong gradient imposes to consider the radial component of the field to account for the transverse variations of the field magnitude. This is were the $S_{n}^{m}$ matrix and the previous results will come in handy.

It is well known that the achievement of high-resolution imaging in a stray field requires to locate the sensitive slice at a particular spot along the axis, where the field is relatively homogeneous in the transverse direction (cancelation of first order transverse derivatives) [23-25]. We will call that spot the "sweet spot". It is important to note that it corresponds to a region where uniformity in the transverse direction is achieved for the field magnitude, but not for the field direction. ${ }^{5}$

The "flat" sensitive slice is obtained when the radial (or transverse) variations of $B_{z}$ are compensating the radial variations of $B_{\rho}$. Eq. (15) provides the values of the SHE of $B_{z}$ necessary to satisfy this condition. In the usual STRAFI situation, only one condition can be satisfied at a time (unless the magnet is designed specifically for it, which is rarely the case). Fig. 5 shows a plot of the evolution of $Z_{2}$ and $\frac{1}{4} \frac{Z_{1}^{2}}{Z_{0}}$ for a finite length solenoid with form factor $\frac{b}{a}=3$ ( $a$ being the radius and $b$ the half-length). The intersection of the two curves corresponds to the satisfaction of the condition on $Z_{2}$, which in turn corresponds to the sweet spot. We can notice that it is just after the inflection point (itself just after the end of the solenoid). This fact is well known and has been noted empirically in the literature several times [23-25].

Before we go any further, we should explicit some criteria to assess the "quality" of a given plane in STRAFI. In what follows, we will consider the "curvature" or "peak-to-peak" deviation of the field from its value on-axis when going away from the axis in a transverse plane. In order to consider quantities relevant to imaging, we convert this field deviation to a position deviation. This is based on the assumption that the position of a point $P(\rho, z)$ in the image is given by $\frac{B_{0}(\rho, z)-B_{0}(0,0)}{G_{z}}$ where $G_{z}$ is the gradient at the origin. Thus, if the field profile is perfect (linear variation along $\mathrm{Oz}$, uniform in transverse directions), the image of a plane remains a plane. Any defect in the field will show up as a "curvature" of the image of a plane. We will also simply refer to the image of a plane as the image-plane (even though it is most often not a plane) and to the plane imaged as the object-plane.

A practical application of the results found in the previous section is that the quality of the sweet spot can be improved by using a shim stack in order to satisfy the conditions on higher degrees such as $Z_{3}, Z_{4}$, and so on. This will have for effect to increase the

\footnotetext{
5 The latter varies actually quite a lot, which may need to be taken into account when designing an excitation and detection device. Otherwise, the excitation and detection efficiency decreases as we go away from the axis, though the $B_{1}$ field is homogeneous.
}

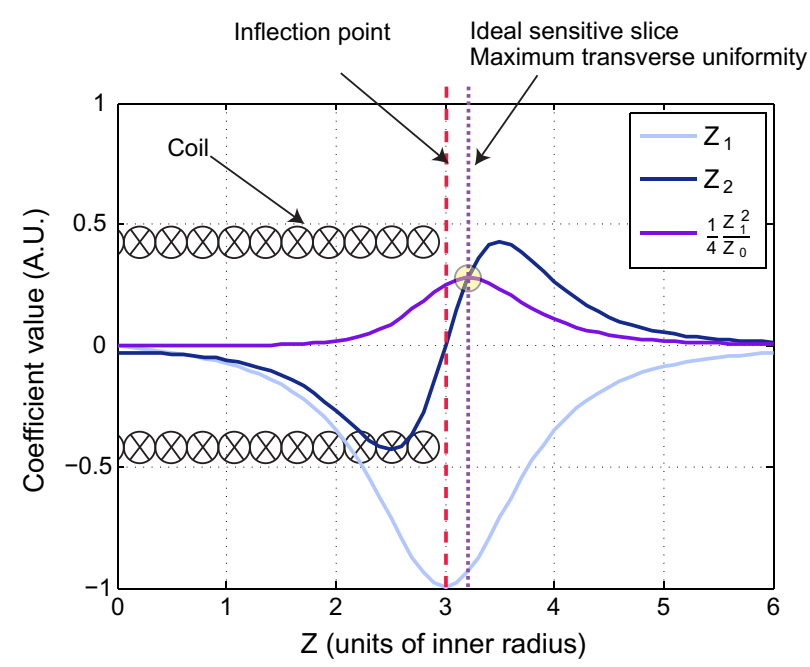

Fig. 5. Evolution of the first SHE terms along the axis for a solenoid of radius one unit and length six units. The end of the coil is shown. The maximum of $Z_{1}$ occurs shortly after the end of the coil. The natural sweet spot, where the field is the most uniform in transverse planes is the plane noted by the circle, where $Z_{2}=\frac{1}{4} \frac{Z_{1}^{2}}{Z_{0}}$. This plane is located outside of the coil, very close to its end, as it has been empirically noticed on superconducting magnets.

extent of the flat slice, or increase the available resolution for a given size. We can again take the example of the thin solenoid. By using a set of current loops, we can generate $Z_{2}, Z_{3}$ and $Z_{4}$ independently and match the conditions on these terms. In the sweet spot, the condition on $Z_{2}$ is already satisfied and only $Z_{3}$ and $Z_{4}$ need to be adjusted. In the simulation used to produce Fig. 6 , we have used a solenoid of radius $100 \mathrm{~mm}$ and length $400 \mathrm{~mm}$, producing a gradient of $60 \mathrm{~T} \mathrm{~m}^{-1}$ with a field of $4.99 \mathrm{~T}$ in the sweet spot, located $221.01 \mathrm{~mm}$ away from the center of the solenoid. The simulated shim coils are sets of simple loops (radius $100 \mathrm{~mm}$ ) centered on the sweet-spot and optimized following the methods found in $[1,3,17]$. The currents in the loops were adjusted in order to satisfy at the same time the conditions on $Z_{2}, Z_{3}$ and $Z_{4}$ in the sweet-spot. The improvement brought by the shim coils to the instrumental resolution is about a factor ten, as seen in Fig. 6.

The improvement of the sweet spot takes another dimension when considering the longitudinal extent of this region. It is first of all interesting to consider the evolution of the image-planes along $\mathrm{Oz}$ around the sweet spot (at elevation $z_{0}$ ) in the "natural" case (i. e. without shimming). It is indeed worthwhile noting that the satisfaction of condition $Z_{2}=\frac{1}{4} \frac{Z_{1}^{2}}{Z_{0}}$ guarantees the highest resolution only when the extent of the object considered is small enough that the contribution of higher degree terms is negligible. Otherwise, the combination of the different terms results in a complicated variation of the image-plane shape. In some cases, the best resolution can be found at a different location from $z_{0}$ (see the evolution of the image-planes with $z$ in Fig. 6). We can go even further by noting that the actual shape of the image-plane in a given location is not as much relevant to the evaluation of the "slice-quality" (image-plane curvature) as its peak-to-peak amplitude. The latter gives us the apparent thickness of an infinitely thin sample, or the ultimate resolution (which could be called instrumental resolution). Based on the previous solenoid configuration, we have computed this instrumental resolution around the sweet-spot for different sample extents, giving valuable quantitative information about what can be expected (see Fig. 7). It is worth noting that the resolution degrades rather quickly without correction. It is thus critical to determine accurately the position of the sweet-spot.

We have only considered a solenoid until now. NMR superconducting magnets are obviously more complicated as they are designed to generate a much more homogeneous field in their cen- 

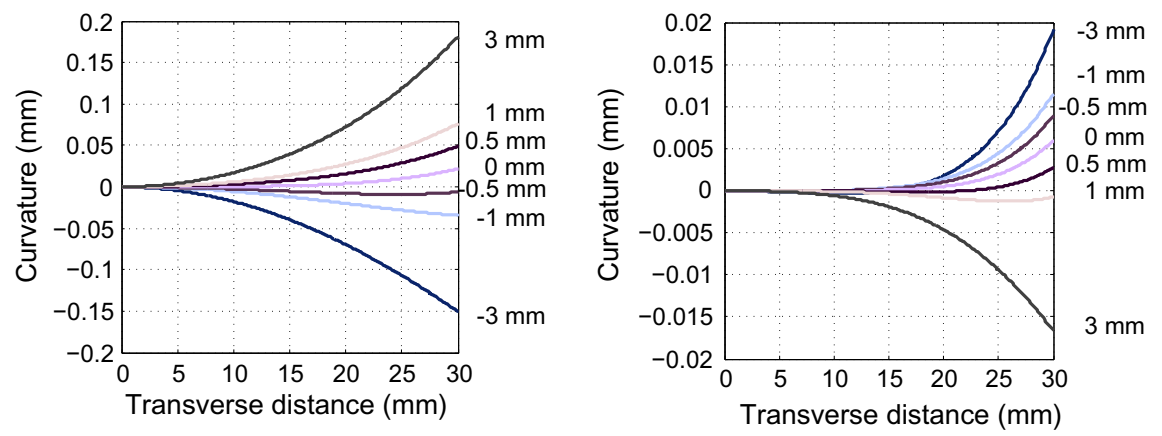

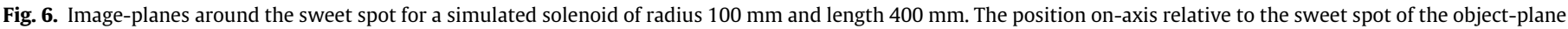

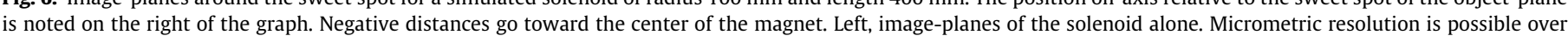

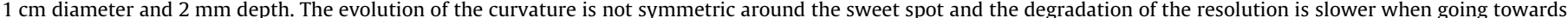

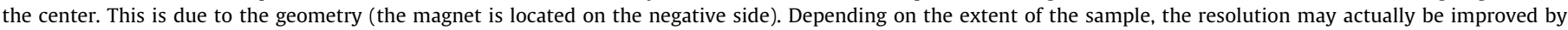

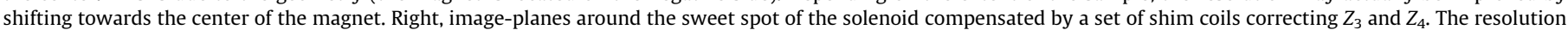
improvement is at least a factor 5 (depending on the position on-axis) and micrometric resolution is possible over $2 \mathrm{~cm}$ diameter and at least $6 \mathrm{~mm}$ depth.
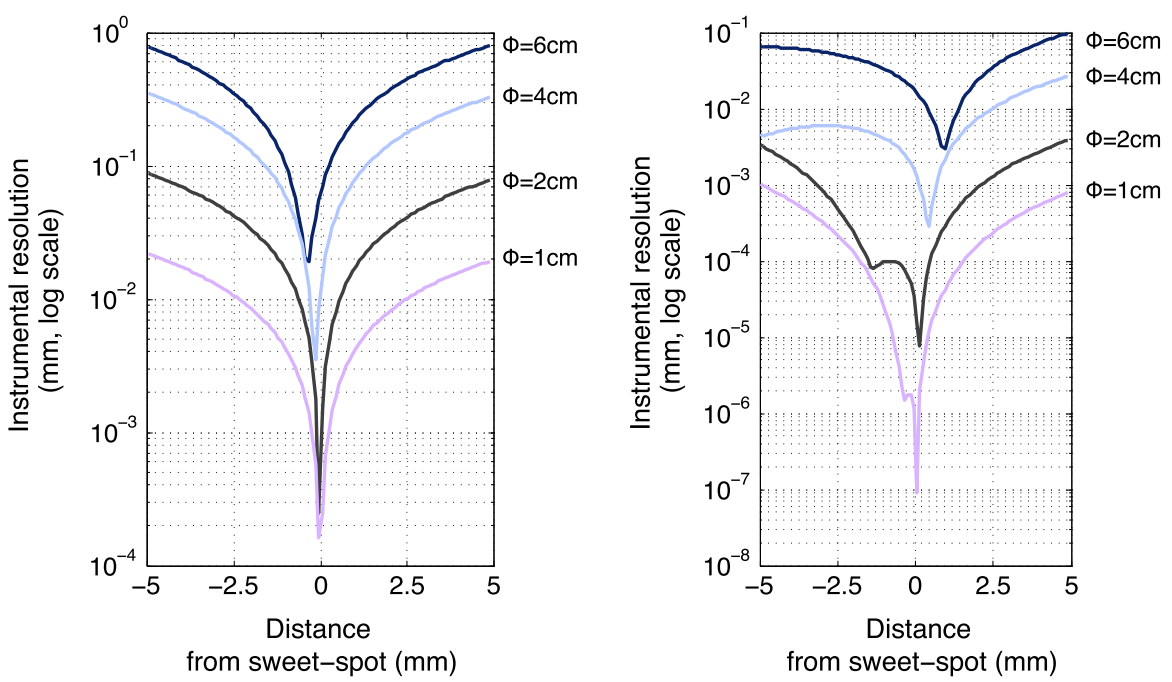

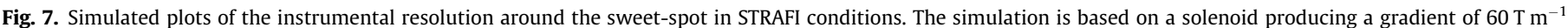

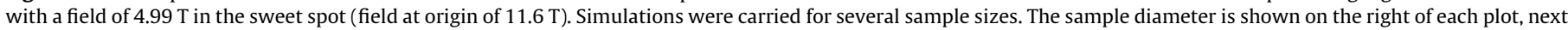

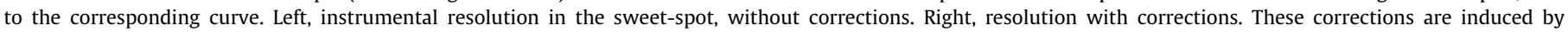
simulated shim coils producing zonal harmonics which satisfy the conditions of Eq. (15). The improvement in resolution is at least a factor ten.

ter. It is thus likely that the location of the sensitive slice will be different, and that the harmonic content of $B_{z}$ will vary differently along $\mathrm{Oz}$. In most cases, the geometry of the magnet is unknown, so that it is impossible to compute a priori the different terms. Thus, any STRAFI experiment requires a search for the natural sweet spot of the magnet. This can present practical difficulties as the detection of a region where the sensitive slice is very flat requires to have a very thin sample with a very well-controlled orientation. In addition, we have just seen that it is critical to locate the sweet-spot accurately (better than $1 \mathrm{~mm}$ ). The previous discussions and results provide an alternative way of locating this natural sweet spot. We have indeed shown that it is sufficient to know the $Z_{n}$ evolution along the axis to be able to locate the sweet spot. The following measurement method provides this information.

The calculation of $N Z_{n}$ terms requires at least to measure the field in $N$ points of elevation $z_{j}$ located on the axis. This can be easily done by sliding a NMR probe with a small sample centered on the axis. A simple polynomial interpolation of these measurements will provide accurate values of $N Z_{n}$ terms (provided it is done appropriately and uncertainties on the measurements are not too great). The best results will be obtained by using the Chebyshev nodes so that the Runge phenomenon is minimized (the $z_{j}$ correspond to the zeros of the Chebyshev polynomial of degree $N$ ) [26]. We can then use the following equation to compute the $Z_{n}^{\prime}$ at a different location on the axis (see demonstration in Appendix C):

$Z_{n}^{\prime}=\sum_{k=n}^{\infty} Z_{k} C_{k}^{n} z_{0}^{k-n}$

This formula provides a way to compute the terms at a location $O^{\prime}$ on the axis distant by $z_{0}$ from the initial origin $O$, based only on the measured terms in $O$. It is thus sufficient to perform the measurements only once, provided enough measurements are taken. The requirement on the number of measurements depends on the convergence of the infinite sum. Here, the domain of convergence corresponds to $z_{0}<R_{0}$, where $R_{0}$ is the radius of the largest sphere centered on $O$ that does not contain any source of magnetic field. In that case, we can truncate the infinite sum in Eq. (28) and compute the expansion coefficients in $O^{\prime}$ with a finite number of terms from the expansion in $O$. Obviously, the speed of convergence of $Z_{n}^{\prime}$ depends on $\frac{z_{0}}{R_{0}}$. The smaller $z_{0}$, the less terms are needed 
to compute $Z_{n}^{\prime}$. In practice, it is thus more effective to perform the initial measurements close to the region we are looking for. This is not difficult as we have already noted that it is nearby the inflection point, a point relatively easy to locate. To give an idea of the precision of this procedure, we performed simulations of such measurements for a solenoid with radius $100 \mathrm{~mm}$ and length $400 \mathrm{~mm}$, generating $11.6 \mathrm{~T}$ (see Fig. 7). It appears that for a resolution of $100 \mathrm{ppm}$ on the measured field values and a resolution of $0.05 \mathrm{~mm}$ on the position of the measurement points, only $12 \mathrm{mea}$ surements are necessary to obtain the position of the sweet spot within $0.14 \mathrm{~mm}$ accuracy and $0.6 \mathrm{~mm}$ precision (see Fig. 8 ). ${ }^{6}$ The accuracy of the position is determined by the number of points (it is a matter of convergence), while the precision is determined by the uncertainties on the field values and the positions. Precision degrades as the number of points increases for given uncertainties on field values and positions. Of course, if the uncertainties on the field values and the positions are better, the precision will also be improved but accuracy will remain about the same (unless using many more points, where the accuracy becomes limited by the precision on the direct measurements). It is important to note that the field resolution might be difficult to achieve, due to the gradient strength. A solution is to use the smallest sample possible and implement a simple gradient compensation coil that moves with the sample, as proposed in [17]. From these estimations, and because the method can be applied without prior knowledge about the magnet, it seems this method is suitable to provide an estimate of the location of the STRAFI sweet spot within $0.5 \mathrm{~mm}$ for any magnet. This method provides thus a direct way to position a $1 \mathrm{~cm}$ diameter sample and obtain potentially micrometer resolution, according to Fig. 7.

While we have only considered the possibility of locating a natural sweet spot, we can go further and create a sweet spot in any arbitrary point on the axis, using a shim system. We just need to apply the appropriate amount of $Z_{2}, Z_{3}, Z_{4}$, and so on, to satisfy the conditions anywhere we choose. This operation can be guided by the results of the previous measurement method (now centered on the spot we are interested in). Let us take the example of the previous solenoid one more time. We can, for example decide to perform the experiment at $z_{0}=100 \mathrm{~mm}$. The gradient is now about $20 \mathrm{~T} \mathrm{~m}^{-1}$ and the conditions from Eq. (15) are not satisfied at all. This results in an important curvature of the sensitive slice, degrading a lot the resolution. By applying the appropriate shims and satisfying Eq. (15), we can improve the resolution by two orders of magnitude, as seen in Fig. 9.

Obviously the arbitrary creation of a sensitive slice will be limited by the strength of the shim system used. For example, the previous example requires to be able to generate about $100 \mathrm{ppm}$ of the main field (at the center of the solenoid) of $Z_{2}$ over a radius of $2.5 \mathrm{~mm}$. It is unlikely that any standard shim stack will be able to generate such an amount of correction. It is probably necessary to combine a standard shim stack for fine adjustment with a set of passive shims which can be calculated using the methods found in [27] for example.

\section{Conclusions and perspectives}

We have introduced a compact and practical framework to describe the variations of $B^{2}$, which reflect the ones of $B$. This framework is particularly suitable when the magnetic field modulus cannot be approximated by only one component of the magnetic field. This is specifically the case when dealing with strong gradients. This framework finds its roots in the usual one used for

\footnotetext{
${ }^{6}$ In a measurement process affected by random noise, we designate the accuracy as the difference between the mean of the measurement outcome and the actual, "true", value. We call precision the interval span by three standard deviations of the distribution resulting from a great number of measurement attempts (typically more than 1000 , until the distribution of results approaches a Gaussian distribution).
}

\begin{tabular}{|c|c|c|c|}
\hline Sample points & Average position $(\mathrm{mm})$ & Accuracy $(\mathrm{mm})$ & Precision $(\mathrm{mm})$ \\
\hline 6 & 237.0 & 16.00 & 0.1 \\
\hline 8 & 224.6 & 3.56 & 0.2 \\
\hline 10 & 221.8 & 0.78 & 0.4 \\
\hline 12 & 221.1 & 0.14 & 0.6 \\
\hline 14 & 221.0 & 0.04 & 1.1 \\
\hline
\end{tabular}

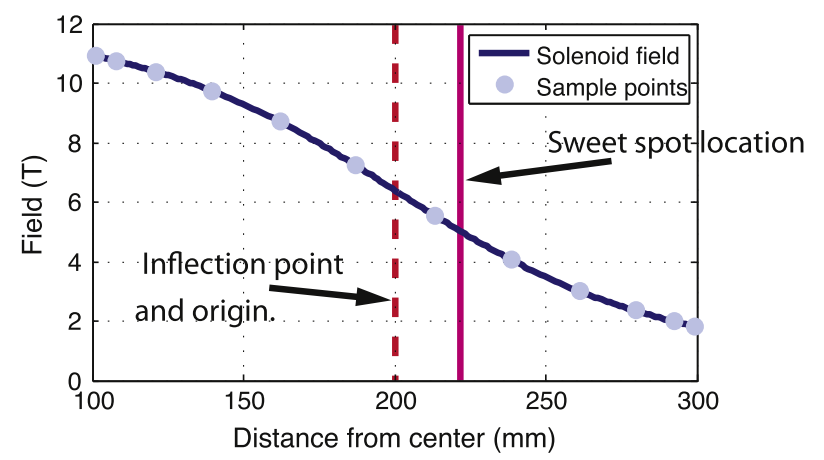

Fig. 8. Estimation of accuracy and precision on the experimental computation of the sweet spot position for STRAFI on the axis of a solenoid, according to the method described in the text. 10,000 measurements were simulated (based on the theoretical field of a solenoid) with uncertainty of $100 \mathrm{ppm}$ on the field values and $0.01 \mathrm{~mm}$ on the point positions. The position is computed based on the coefficients determined by polynomial interpolation of the sample points, Eqs. 28 and 15. The average value is given in the table, along with the accuracy (difference between average value and actual sweet spot position), and the precision (spread of the distribution given by 3 sigma). The plot shows an example of sample points for $N=10$. The accuracy is almost entirely determined by the number of sample points used. Precision is mostly determined by uncertainties on the quantities measured directly (field values, positions) and is degraded by increased number of points. This latter effect is due to the small number of points used. The effect on precision will stabilize as the number of points grows. However, when using less than 20 points, it appears important to make a compromise between accuracy and precision.

homogeneous fields so that it can be easily integrated with current approaches and devices. We proposed three examples where this framework finds an application, namely permanent magnet design, field plotting, and STRAFI NMR.

While we demonstrated its effectiveness for the design of permanent magnets generating gradients, it is obvious that this method can be used to design axial coils generating $\frac{\partial B}{\partial z}$. However, the assumption of cylindrical symmetry prevents from using this framework for transverse gradients $\left(\frac{\partial B}{\partial x}, \frac{\partial B}{\partial y}\right)$ unless the geometry allows to keep cylindrical symmetry for these gradients (it is generally not the case). The framework could be generalized to arbitrary situations without symmetry but the resulting analytical expressions become rapidly difficult to manage.

Though we focused on NMR applications, it is important to note that strong gradients with well-controlled profiles can be used for imaging modalities such as Magnetic Particle Imaging (MPI) instruments [28], or for diamagnetic levitation in the design of magnetic traps $[29,30]$.

Another case where the results of this study may be useful is STRAFI and STRAFI-MAS imaging, where the sample is rotated to accomplish 3D images using only the stray field gradient [31]. In that case, distortions due to the non-linearity of the field variations are observed [32]. Here again, this approach could be used to correct for undesired field variations. Furthermore this approach could lead to dedicated superconducting magnets where the sweet spot conditions described in this paper could be satisfied by controlling the cryo-shims, providing thus extended volume linear gradient at the center of the magnet. This would be very useful for all STRAFI experiments since the probe would not need to be tuned to lower frequencies, an operation that can lead to suboptimal performance. 

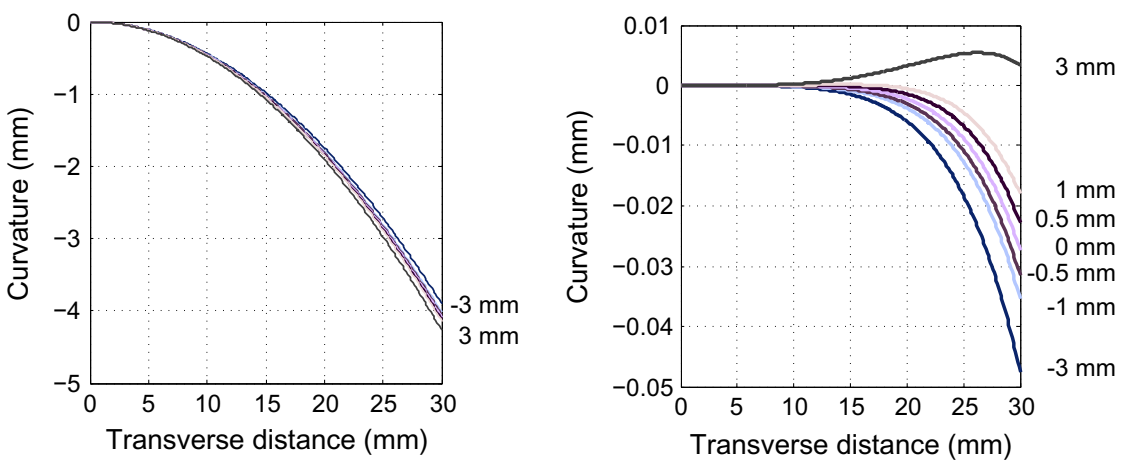

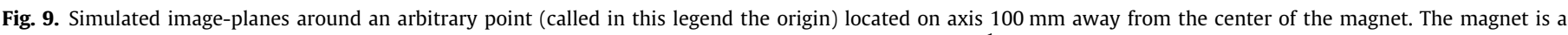

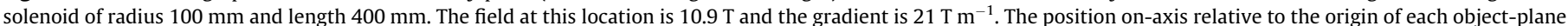

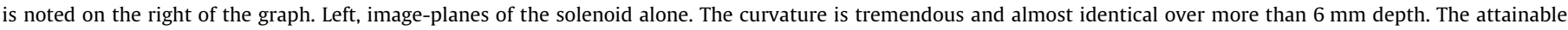

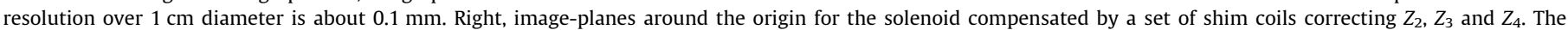

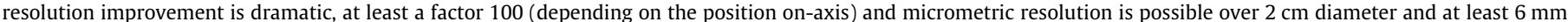

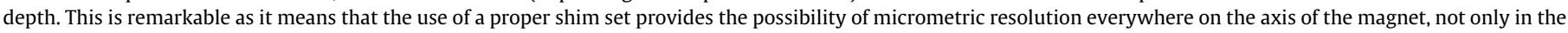
sweet spot region.

\section{Acknowledgments}

The authors would like to thank Dr. J. Baltisberger and Dr. A. Wong for critical reading and comments about the manuscript. The research leading to these results has received funding from the European Research Council under the European Community's Seventh Framework Programme (FP7/2007-2013): ERC Grant Agreement No. 205119 and partially by the C'Nano IdF grant "nanoMACS".

\section{Appendix A. Derivation of the expansion of $B_{\rho}$}

Following the notation used in $[2,3,9]$, we can write the general form of the SHE for the scalar potential $\Phi^{*}$ with cylindrical symmetry around $O z$, using spherical coordinates $(r, \phi, \theta)$, as

$\Phi^{*}(r, \theta)=\sum_{n=0}^{\infty} \bar{Z}_{n} r^{n} P_{n}(\cos \theta)$

and for $B_{z}$

$B_{z}(r, \theta)=\sum_{n=0}^{\infty} Z_{n} r^{n} P_{n}(\cos \theta)$

with the $P_{n}$ as the Legendre polynomials and

$Z_{n}=-(n+1) \bar{Z}_{n+1}$

We can in addition derive the one of $B_{\rho}$. We have indeed

$B_{\rho}=-\frac{\partial \Phi^{*}}{\partial \rho}$

and

$\frac{\partial}{\partial \rho}=\sin \theta \frac{\partial}{\partial r}+\frac{\cos \theta}{r} \frac{\partial}{\partial \theta}$.

If we take now only one term of degree $n$ in the potential, we have

$$
\begin{aligned}
A & =\frac{\partial}{\partial \rho}\left(r^{n} \bar{Z}_{n} P_{n}(\cos \theta)\right) \\
& =\sin \theta n r^{n-1} \bar{Z}_{n} P_{n}(\cos \theta)+\frac{\cos \theta}{r} r^{n} \bar{Z}_{n} \frac{d P_{n}(\cos \theta)}{d \theta} .
\end{aligned}
$$

In the meanwhile we have [33]

$P_{n}^{1}(x)=\sin \theta \frac{d P_{n}(x)}{d x}$.
Consequently,

$A=r^{n-1} \bar{Z}_{n}\left(n \sin \theta P_{n}(\cos \theta)-\cos \theta P_{n}^{1}(\cos \theta)\right)$.

We can now use a few well-known relations between Legendre polynomials of different degrees and orders [1] so that we can write

$\frac{\partial}{\partial \rho}\left(r^{n} \bar{Z}_{n} P_{n}(\cos \theta)\right)=-\bar{Z}_{n} r^{n-1} P_{n-1}^{1}(\cos \theta)$.

Thus, using Eq. (31), we have

$$
\begin{aligned}
& B_{\rho}=\sum_{n=1}^{\infty}-\frac{1}{n+1} Z_{n} r^{n} P_{n}^{1}(\cos \theta), \\
& B_{z}=Z_{0}+\sum_{n=1}^{\infty} Z_{n} r^{n} P_{n}(\cos \theta) .
\end{aligned}
$$

\section{Appendix B. Derivation of the analytical formulae of $Z_{n}$ for an axially magnetized hollow cylinder}

This ring is equivalent to the superposition of two solid cylinders of radius $a_{1}$ and $a_{2}$ and delimited by the same planes as the ring. Cylinder 2 is magnetized in the same direction as the ring and cylinder 1 is magnetized in the opposite direction. Such cylinders are equivalent to solenoids of same radii and height with surface current density equal to $M$ and $-M$ respectively. The field generated on the axis by a solenoid of finite length is well known and can be written [22] as

$B_{z_{\text {sol }}}(r=0, z)=\frac{\mu_{0} M}{2}\left[\frac{b-z}{\sqrt{a^{2}+(b-z)^{2}}}\right]_{b_{1}}^{b_{2}}$.

The brackets $[f(b)]_{b_{1}}^{b_{2}}$ are a short notation for $f\left(b_{2}\right)-f\left(b_{1}\right)$. Hence, the field generated by the ring is given by

$B_{z}(r=0, z)=\frac{\mu_{0} M}{2}\left[\left[\frac{b-z}{\sqrt{a^{2}+(b-z)^{2}}}\right]_{a_{1}}^{a_{2}}\right]_{b_{1}}^{b_{2}}$

Thus,

$Z_{0}=\frac{\mu_{0} M}{2}\left[\left[\frac{b}{\sqrt{a^{2}+b^{2}}}\right]_{a_{1}}^{a_{2}}\right]_{b_{1}}^{b_{2}}=\frac{\mu_{0} M}{2}\left[[\cos \alpha]_{a_{1}}^{a_{2}}\right]_{b_{1}}^{b_{2}}$, 
with

$a=c \sin \alpha$,

$b=c \cos \alpha$

$c=\sqrt{a^{2}+b^{2}}$.

A term of degree $N+1$ can be derived from the term of degree $N$ thanks to the following relation (simple differentiation)

$Z_{n}=-\frac{1}{n}\left[\left[\frac{\partial Z_{n-1}}{\partial b}\right]_{a_{1}}^{a_{2}}\right]_{b_{1}}^{b_{2}}$

After applying this relation, we find

$Z_{1}=-\frac{\mu_{0} M}{2}\left[\left[\frac{1}{c} \sin \alpha P_{1}^{1}(\cos \alpha)\right]_{a_{1}}^{a_{2}}\right]_{b_{1}}^{b_{2}}$.

However, if we look at the derivative of $\frac{1}{c^{n}} \sin \alpha P_{n}^{1}(\cos \alpha)$, we get

$\frac{\partial}{\partial b}\left(\frac{1}{c^{n}} \sin \alpha P_{n}^{1}(\cos \alpha)\right)=-\frac{n}{c^{n+1}} \sin \alpha P_{n+1}^{1}$

Thus, by induction, we find that

$Z_{n \geqslant 1}=-\frac{\mu_{0} M}{2 n}\left[\left[\frac{1}{c^{n}} \sin \alpha P_{n}^{1}(\cos \alpha)\right]_{a_{1}}^{a_{2}}\right]_{b_{1}}^{b_{2}}$.

\section{Appendix C. Derivation of Eq. (28)}

We simply need to note that the field on axis is a simple expansion in powers of $z$. As a result, knowing the expansion at one point of the axis, we can compute the expansion in any other point, provided we know enough terms. Let us define an origin $O$ and another one $O^{\prime}$. They are both on axis and $O^{\prime}$ is at position $z_{0}$ compared to $O$. We note $z$ the position of the evaluation point relative to $O$ and $\zeta$ the position relative to $O^{\prime}$. Thus $z=\zeta+z_{0}$. We have in $O$ the expansion

$B_{z}(z)=\sum_{n=0}^{\infty} Z_{n} z^{n}$

We have thus

$B_{z}(z, \zeta, z 0)=\sum_{n=0}^{\infty} Z_{n} \sum_{k=0}^{n} C_{n}^{k} z_{0}^{n-k} \zeta^{k}$

which can be transformed into an expansion in terms of $\zeta$

$B_{z}\left(z^{\prime}\right)=\sum_{k=0}^{\infty} \sum_{n=k}^{\infty} Z_{n} C_{n}^{k} z_{0}^{n-k} \zeta^{k}$

where the $C_{n}^{k,}$ s are the binomial coefficients.

If we write in $O^{\prime}$,

$B_{z}(\zeta)=\sum_{p=0}^{\infty} Z_{p}^{\prime} \zeta^{p}$

we can identify the $Z_{p}^{\prime}$ using Eq. (50), so that

$Z_{p}^{\prime}=\sum_{k=p}^{\infty} Z_{k} C_{k}^{p} z_{0}^{k-p}$

\section{References}

[1] F. Roméo, D.I. Hoult, Magnet field profiling: analysis and correcting coil design, Magnetic Resonance in Medicine 1 (1984) 44-65.

[2] C. Hugon, P.M. Aguiar, G. Aubert, D. Sakellariou, Design, fabrication and evaluation of a low-cost homogeneous portable permanent magnet for NMR and MRI, Comptes Rendus de Chimie de l'Académie des Sciences 13 (2010) 388.

[3] C. Hugon, F. D’Amico, G. Aubert, D. Sakellariou, Design of arbitrarily homogeneous permanent magnet systems for NMR and MRI: theory and experimental developments of a simple portable magnet, Journal of Magnetic Resonance 205 (2010) 75.

[4] R. Kimmich, NMR, Tomography, Diffusometry, Relaxometry, Springer, 1997.

[5] A. Samoilenko, D.Y. Artemov, L. Sibel'dina, Formation of sensitive layer in experiments on NMR subsurface imaging of solids, JETP Letters 47 (7) (1988) 417-419.

[6] B. Blümich, P. Blümler, G. Eidmann, A. Guthausen, R. Haken, U. Schmitz, K. Saito, G. Zimmer, The NMR-MOUSE: construction, excitation, and applications, Magnetic Resonance Imaging 16 (5/6) (1998) 479-484.

[7] P. Glover, P. Aptaker, J. Bowler, E. Ciampi, P. McDonald, A novel high-gradient permanent magnet for the profiling of planar films and coatings, Journal of Magnetic Resonance 139 (1999) 90-97.

[8] P. McDonald, P. Aptaker, J. Mitchell, M. Mulheron, A unilateral NMR magnet for sub-structure analysis in the built environment: the surface GARField, Journal of Magnetic Resonance 185 (2007) 1-11.

[9] C. Hugon, G. Aubert, D. Sakellariou, A systematic approach to the design, fabrication and testing of permanent magnets applied to single-sided NMR, in: AIP Conference Proceedings, vol. 1330, 2011, pp. 97-100.

[10] D.G. Norris, J.M. Hutchison, Concomitant magnetic field gradients and their effects on imaging at low magnetic field strength, Magnetic Resonance Imaging 8 (1) (1990) 33-37.

[11] M.A. Bernstein, X.J. Zhou, J.A. Polzin, K.F. King, A. Ganin, N.J. Pelc, G.H. Glover Concomitant gradient terms in phase contrast MR: analysis and correction, Magnetic Resonance in Medicine 39 (1998) 300-306.

[12] C.A. Meriles, D. Sakellariou, A.H. Trabesinger, V. Demas, A. Pines, Zero- to lowfield MRI with averaging of concomitant gradient fields, PNAS 102 (6) (2005) 1842-1844.

[13] C.A. Meriles, D. Sakellariou, A.H. Trabesinger, Theory of MRI in the presence of zero to low magnetic fields and tensor imaging field gradients, Journal of Magnetic Resonance 182 (2006) 106-114.

[14] T. Classen-Vujcic, J. Slotboom, A.F. Mehlkopf, Reduction of concomitant field gradient effects by main field alteration, in: Proceedings SMRM, 1995, p. 315.

[15] T. Classen-Vujcic, K. Blom, A.F. Mehlkopf, Hardware Corrections of concomitant field gradients, in: Proceedings of the SMRM, 1997, p. 56.

[16] É. Durand, Magnétostatique, Masson, 1968. p. 27.

[17] C. Hugon, Aimants permanent pour la RMN et l'IRM/permanent magnets for NMR and MRI (text in english), Ph.D. thesis, Université de Versailles-SaintQuentin-en-Yvelines, October 2010.

[18] M. Van Landeghem, B. Bresson, B. Blümich, J. d'Espinose de Lacaillerie, Micrometer scale resolution of materials by stray-field magnetic resonance imaging, Journal of Magnetic Resonance 211 (2011) 60-66.

[19] S. Anferova, V. Anferov, M. Adams, P. Blümler, N. Routley, K. Hailu, K. Kupferschläger, M. Mallett, G. Schroeder, S. Sharma, B. Blümich, construction of a NMR-MOUSE with short deat time, Concepts in Magnetic Resonance 14 (1) (2002) 15-25.

[20] A.E. Marble, I.V. Mastikhin, B.G. Colpitts, B.J. Balcom, Designing static fields for unilateral magnetic resonance by a scalar potential approach, IEEE Transactions on Magnetics 43 (5) (2007) 1903-1911.

[21] J.L. Paulsen, L.S. Bouchard, D. Graziani, B. Blümich, A. Pines, Volume-selective magnetic resonance imaging using an adjustable, single-sided, portable sensor, PNAS 105 (52) (2008) 20601-20604.

[22] É. Durand, Magnétostatique, Masson, 1968

[23] E.W. Randall, Stray field (STRAFI) NMR: imaging in large field-gradients, Encyclopedia of Magnetic Resonance (2011)

[24] P. McDonald, B. Newling, Stray field magnetic resonance imaging, Reports on Progress in Physics 61 (1998) 1441-1493.

[25] P. Glover, P.J. McDonald, B. Newling, Stray-field imaging of planar films using a novel surface coil, Journal of Magnetic Resonance 126 (1997) 207-212.

[26] J.P. Boyd, Chebyshev and Fourier Spectral Methods, Dover Publications, 2001

27] D. Hoult, D. Lee, Shimming a superconducting nuclear magnetic resonance imaging magnet with steel, Review of Scientific Instruments 56 (1) (1985) 131-135.

[28] B. Gleich, J. Weizenecker, Tomographic imaging using the nonlinear response of magnetic particles, Nature 435 (30) (2005) 1214-1217.

[29] H. Chetouani, C. Jeandey, V. Haguet, H. Rostaing, C. Dieppedale, G. Reyne Diamagnetic levitation with permanent magnets for contacless guiding and trapping of microdroplets and particles in air and liquids, IEEE Transactions on Magnetics 42 (10) (2006) 3557-3559.

[30] H. Chetouani, C. Jeandey, V. Haguet, H. Rostaing, C. Dieppedale, J.-F. Jacquot, T. Kerlin, G. Reyne, Principle and analysis of a two-dimensional onchip magnetophoresis of bioparticles for contamination-free biochemical reactors, IEEE Transactions on Magnetics 43 (4) (2007) 1673-1676.

[31] J.H. Baltisberger, S. Hediger, L. Emsley, Multi-dimensional magnetic resonance imaging in a stray magnetic field, Journal of Magnetic Resonance 172 (2005) 79-84.

[32] A. Wong, D. Sakellariou, Two- and three-dimensional multinuclear stray-field imaging of rotating samples with magic-angle spinning (STRAFI-MAS): from bio to inorganic materials, Journal of Magnetic Resonance Imaging 32 (2010) 418-423.

[33] G.B. Arfken, H.J. Weber, Mathematical Methods for Physicists, sixth ed. Elsevier Academic Press, 2005. 